\title{
Une nouvelle image des Pays-Bas : l'identité nationale par l'estampe
}

\section{Eveline Koolhaas-Grosfeld}

\author{
(2) OpenEdition \\ Journals \\ Édition électronique \\ URL : https://journals.openedition.org/ahrf/523 \\ DOI : $10.4000 / a h r f .523$ \\ ISSN : 1952-403X \\ Éditeur : \\ Armand Colin, Société des études robespierristes \\ Édition imprimée \\ Date de publication : 1 décembre 2001 \\ Pagination : 141-159 \\ ISSN : 0003-4436 \\ Référence électronique \\ Eveline Koolhaas-Grosfeld, "Une nouvelle image des Pays-Bas : l'identité nationale par l'estampe », \\ Annales historiques de la Révolution française [En ligne], 326 I octobre- décembre 2001, mis en ligne le \\ 21 mars 2008, consulté le 24 avril 2022. URL : http://journals.openedition.org/ahrf/523 ; DOI : https:// \\ doi.org/10.4000/ahrf.523
}




\title{
UNE NOUVELLE IMAGE DES PAYS-BAS : L'IDENTITÉ NATIONALE PAR L'ESTAMPE
}

\author{
EVELINE KOOLHAAS-GROSFELD
}

\begin{abstract}
À la lumière d'une publication illustrée des années 1803-1807, l'auteur s'interroge ici sur la signification de la revalorisation des costumes nationaux. Dans ces années-là, en effet, un éditeur néerlandais s'avise de publier une série de gravures consacrées aux costumes des diverses provinces bataves. L'entreprise qui vise entre autres à souligner la richesse et l'ancienneté de ces costumes rencontre un grand succès. La question est de savoir ce que cette initiative révèle de l'identité nationale et en quoi elle tente de raviver le sentiment national, blessé par les traumatismes engendrés par la période francobatave.
\end{abstract}

Mots clés : costumes ; habillement ; ethnographie ; République batave ; identité nationale.

« Prospectus des costumes, des mœurs et des coutumes de notre patrie au début du XIXe siècle, dont le premier cahier paraît dès à présent [..:] et qui sera complété par quatre livraisons à paraître tous les trois mois. Chaque planche in-quarto avec explication contiendra deux images «en action», dessinées d'après nature et en couleurs [...] L'éditeur est convaincu que cet ouvrage incomparable par sa beauté et le soin avec lequel il a été exécuté, surpassera de loin celui de la Suisse, ou de tout autre paru jusqu'ici; il n'est pas seulement intéressant en tant que tableau parlant de notre Patrie, mais on y voit encore une grande diversité, inconnue des autres Pays, et ce, sur un si petit territoire, le tout [...] est édité par E. Maaskamp [...] éditeur à Amsterdam. »

C'est ainsi que l'éditeur Evert Maaskamp annonçait dans le Leydsche Courant du 9 mai 1803 la parution d'une série inédite de gravures représentant les différents costumes régionaux de la République batave (illustration 1). Le quatrième cahier parut en 1805 , et deux ans plus tard il fut suivi d'un cinquième; ce qui fait au total vingt images qui sont toutes accompagnées d'un commentaire en néerlandais et en français. En 1805, une intro- 


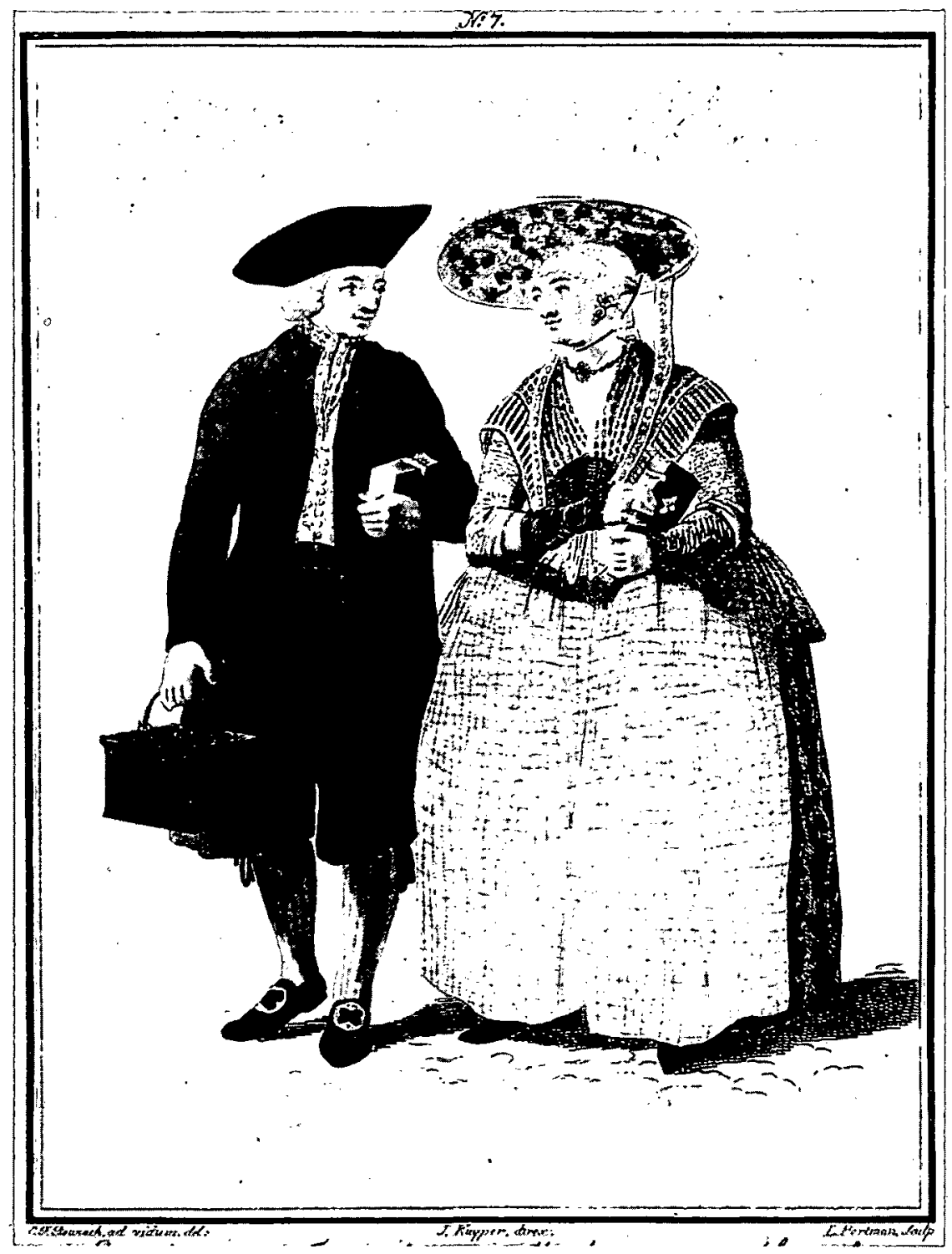

\section{Illustration 1}

L. Portman d'après C. F. Bounach et J. Kuyper.

«Et wier kood ine Tjerke Heit! Het was koud in de Kerk, vader!

/ Mon homme, comme il faisoit froid au sermon! " (Paysan frison et paysanne).

Dans E. MAASKAMP ET J. KUYPER, Tableau de l'habillement [...], Amsterdam/Londen 1804. La Haye, Koninklijke Bibliotheek. [1374A98-pl. 7] 
duction et un frontispice allégorique complétèrent la série, facilitant la reliure de l'ensemble. Le titre définitif devint: Afbeeldingen van kleeding, zeden en gewoonten in de Bataafsche Republiek met den aanvang der negentiende eeuw, et en français: Tableaux de l'habillement, des moeurs et des coutumes dans la République batave au commencement du dix-neuvième siècle.

Les frontispices des éditions postérieures s'adaptèrent aux changements politiques : la « République batave " se transforma successivement en «Royaume de Hollande» et en «Provinces Septentrionales du Royaume des Pays-Bas " (1).

L'édition de Maaskamp ne fait certainement pas mauvaise figure parmi les albums comparables qui virent le jour en France, en Angleterre, en Allemagne et en Suisse dans les années 1800. Que Maaskamp mentionne expressément la Suisse dans son annonce n'est pas étonnant, puisque les 25 gravures des Costumes de la Suisse sont la référence par excellence pour ce qui est de ce genre de publications (2).

Dans le cadre européen, le livre de Maaskamp ne présente donc point un cas isolé. Il paraît à une époque où les intellectuels découvraient la culture populaire et y consacraient des études sérieuses. Le nouvel intérêt pour l'ethnographie coïncidait dans beaucoup de cas avec la construction d'une identité nationale. Le désir de distinguer sa propre nation des autres, était lui-même une réaction à la tendance générale à l'internationalisation - et à l'hégémonie française - dans les domaines de la politique, de la langue, des arts et de la mode (3). De plus, cette tendance stimulait la compétition artistique et commerciale entre les nations, comme le démontre si bien le texte de l'annonce de Maaskamp. Cependant, en éditant les gravures en collaboration avec la maison d'édition anglaise de Colnaghi \& Co., Maaskamp s'assurait d'une diffusion internationale.

De nos jours, aux Pays-Bas, le costume régional ne se voit qu'au musée. En tant qu'image de publicité, décorant les souvenirs et les dépliants touristiques, il semble par contre être indestructible. Évidemment la représentation s'en est depuis longtemps si simplifiée qu'il a été réduit au statut de cliché. C'est pourquoi il est tellement difficile de s'imaginer qu'il y ait eu un temps où devait être découvert le costume régional en tạt que symbole de

(1) En 1823, parut un sixième cahier. La dernière édition par Maaskamp lui-même date de 1829 , mais les planches furent encore employées après sa mort. Voir pour les différentes éditions de tableaux, W. J. A. ARNTZ, «Maaskamp's book of costume-prints », Quaerendo, 1971, I, pp. 9-12. Id., Quaerendo, 1975, II, pp. 263-264. Des sommaires du fonds de Maaskamp se trouvent dans la Nederlandse bibliografie 1801-1832, pp. 692-695 et dans J. LANDWEHR, Studies on Dutch books with coloured plates published 16621875. Natural history, topography and travel, costumes and uniforms, La Haye, 1976, pp. 57-62.

(2) Il s'agit d'estampes coloriées d'après des dessins de F. N. König, parues en 1801-1802. Voir E. NIENHOLDT et G. WAGNER-NEUMANN, Katalog der Lipperheideschen Kostümbibliothek, Berlin, 1965, $\mathrm{n}^{\circ} \mathrm{Ga} 19$.

(3) P. BURKE, Popular culture in early modern Europe, New York, 1978. Sur la notion d'identité nationale, J. TH. LEERSSEN, «Over nationale identiteit », Theoretische Geschiedenis, XI, 1988, n 4, pp. 417-430. 
l'identité néerlandaise. L'édition des Tableaux sur l'habillement de Maaskamp, nous fait assister à l'éclosion de ce moment historique.

L'intérêt ethnographique pour l'habillement existait certes auparavant, comme en témoignent les livres de costumes du XVIe siècle et les bordures des cartes géographiques du XVIIe siècle. Mais ici les paysans et les pêcheurs se trouvent toujours en compagnie de marchands et d'aristocrates. La gravure autonome ne faisait pas grand cas de l'habillement caractéristique. Le classicisme, qui ne tient compte que de l'universel, s'y opposait. Vers 1800 le point de vue commença à changer. Les livres de modèles pour les artistes et pour les amateurs - un genre de cours de dessin destiné à l'usage privé - expriment un nouvel intérêt pour le sujet (illustration 2). Mais l'habillement caractéristique d'un lieu ou d'une région est rare dans ces séries d'images et une explication élaborée fait défaut.

Dans sa Natuurlijke historie van Holland, parue à partir de 1769, Johannes le Francq van Berkheij, appelé parfois le premier ethnologue néerlandais, donnait beaucoup d'attention à l'habillement et aux mœurs des

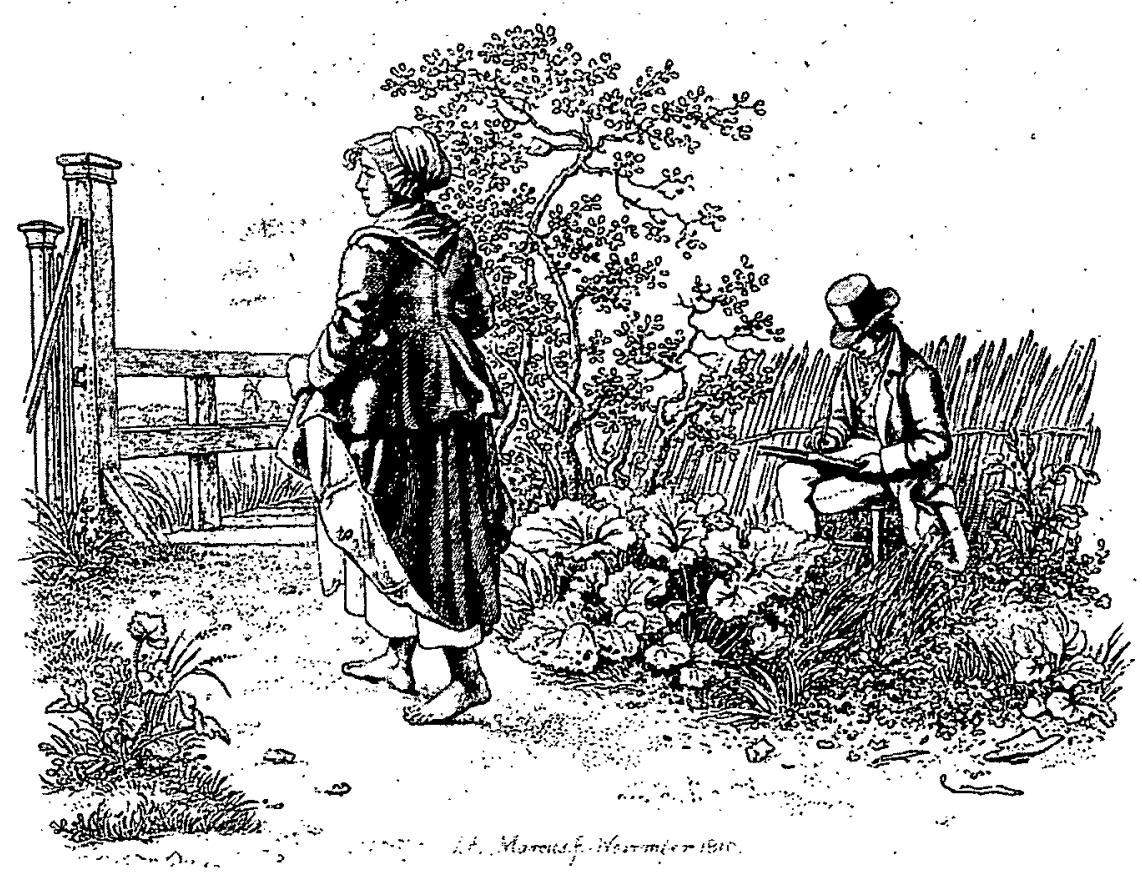

Illustration 2

J. E Marcus, «Een landmeisje en een zittende tekenaar », 1810. (Jeune paysanne et dessinateur assis). Eau-forte dans J. E. MARCUS, Studie-prentwerk, Amsterdam, 1807-1816. Amsterdam, Universiteitsbibliotheek. 
différents ordres au travers des siècles (4). Mais son livre se restreint à la province de Hollande (illustration 3). D'une manière comparable, Adriaan Loosjes présentait l'habillement, connu depuis toujours, de Hindelopen, Molkwerum et Zaandam qu'il montre une fois de plus dans sa revue Kabinet van mode en smaak (1791-1794). Le commentaire accompagnant les gravures selon lequel ces costumes seraient en train de disparaître rapidement, est assez remarquable. En outre, ce ne sont point des images pour attirer les touristes.

Après les cartographes du XVIIe siècle, Maaskamp et ses collaborateurs ont donc été les premiers à approcher de nouveau les costumes régionaux du point de vue de l'ensemble de la nation, en se restreignant pourtant au vêtement des paysans, des pêcheurs et des petits bourgeois. Ils voyaient l'intérêt d'aborder le sujet de façon autonome et conféraient du poids à leur ouvrage en le décrivant poétiquement dans leur annonce comme : «un tableau parlant de la patrie ». Apparemment les créateurs considéraient leurs gravures comme un portrait de groupe qui figurait plus que la somme des diverses parties du pays : c'était la représentation de l'ensemble des Pays-Bas.

La construction de l'image nationale suit les vicissitudes de l'histoire. Vers 1600 , c'était le lion courageux qui représentait les Pays-Bas; au XVII e siècle la vache grasse; au XVIIIe siècle le gros Hollandais, buveur et fumeur (dans les caricatures anglaises du moins) (5). Peu après 1800, l'histoire nationale par l'image adopte un autre cours et réserve une place importante à la femme du peuple. Ce n'est pas un hasard si cette image apparaît justement à une date où les Pays-Bas ont à affronter des problèmes d'image de marque. Attaquant les caprices de la mode, des esprits critiques s'étaient demandé où était passé le «visage national ". Les Français, les Anglais, tous les peuples de l'Europe ont leur costume particulier, écrit le pasteur Ysbrand van Hamelsveld avec un enthousiasme patriote, mais nous, les Néerlandais, nous suivons toutes les modes étrangères (6). Or, ce que Maaskamp allait leur montrer, démentirait ces propos alarmants.

(4) W. FRIJHOFF, "Het zelfbeeld van de Nederlander in de achttiende eeuw: een inleiding", Documentatieblad Achttiende Eeuw, XXIV, 1992, n 1, pp. 5-28. Idem, «Volkskundigen vóor de volkskunde? ", dans TH. DEKKER, P. POST et H. ROODENBURG (éd.), Antiquaren, liefhebbers en professoren, Amsterdam, 1994, pp. 245-267.

(5) B. KEMPERS, « Assemblage van de Nederlandse Leeuw. Politieke symboliek in heraldiek en verhalende prenten uit de zestiende eeuw » dans B. KEMPERS (éd.), Openbaring en bedrog. De afbeelding als historische bron in de Lage Landen, Amsterdam, 1995. M. MEIJER DREES, Andere landen, andere mensen. De beeldvorming van Holland versus Spanje en Engeland omstreeks 1650, La Haye, 1997. N. C. F. VAN SAS, « Eigen en vreemd. Internationale verhoudingen en prentcultuur rondom 1800 », dans B. Kempers, op. cit.

(6) Y. VAN HAMELSVELD, De zedelijke toestand der Nederlandsche natie, Amsterdam, 1791, pp. 289-290. N. C. F. vaN SAS, «De vaderlandse imperatief », dans N. C. F. van Sas (éd.), Vaderland. Een geschiedenis vanaf de vijftiende eeuw tot 1940, Amsterdam, 1999, pp. 298-290. 
146

EVELINE KOOLHAAS-GROSFELD

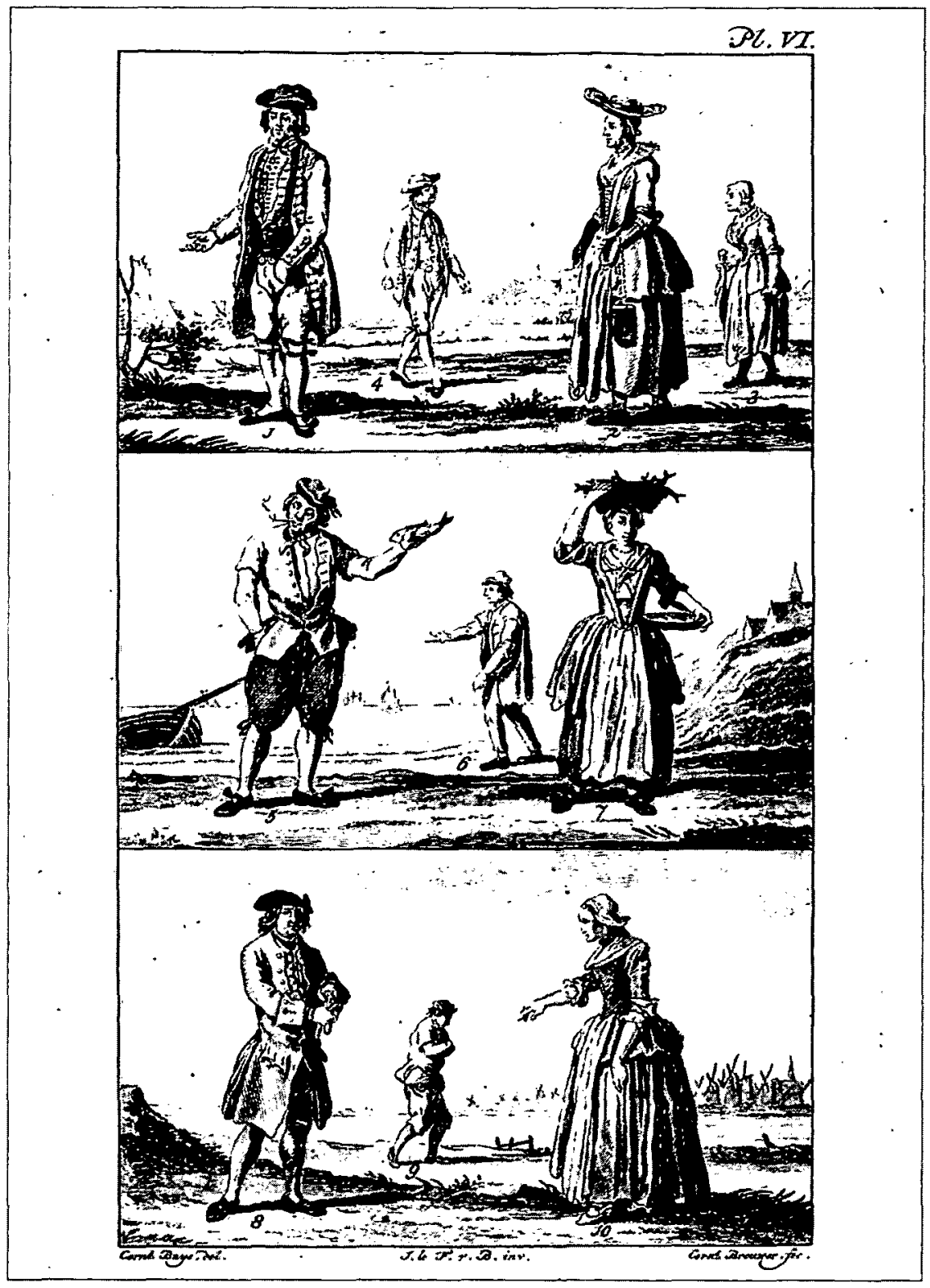

Illustration 3

C. Brouwer d'après J. le Francq van Berkheij. « Hedendaagse deftige boerenstand » (Des paysans distingués contemporains, en haut); « Strandbewoners en zeelieden " (Habitants de la côte et marins, au milieu); "Zaan - en Waterlanders uit de betere Kringen » (Habitants de Zaanland et Waterland aisés, en bas). Dans J. LE FRANCQ VAN BERKHEIJ, Natuurlijke historie van Holland, tome III, Amsterdam 1776. La Haye, Koninklijke Bibliotheek. 


\section{L'éditeur Evert Maaskamp, 1800-1805}

Evert Maaskamp (1769-1834) était marchand de tableaux et éditeur de livres et d'estampes (7). Pour le contenu du fonds, l'éditeur misait sur l'actualité ; pour l'exécution des ouvrages, il visait la meilleure qualité possible et les techniques les plus avancées. Il avait la ferme intention de se mesurer avec la gravure étrangère. Et, dans ce domaine, c'étaient surtout les artistes anglais qui donnaient alors le ton en Europe, grâce à leur perfection inouie dans des techniques comme la demi-teinte, le pointillé (ou l'aquatinte). Dans sa boutique, Maaskamp offrait un riche assortiment de gravures anglaises et françaises. Aux Pays-Bas, un seul artiste pouvait se mesurer avec les Anglais dans le domaine de la gravure : c'était le fameux portraitiste et graveur Charles Howard Hodges, anglais de naissance, que les amateurs néerlandais considéraient volontiers comme un artiste du pays (8).

Maaskamp pouvait se féliciter d'avoir eu Hodges à ses côtés dès le début de sa carrière. Il était assuré non seulement de la qualité des gravures, mais encore de ce qu'elles seraient signées d'un nom anglais. Ses premières éditions sont ainsi presque toutes des demi-teintes gravées par Hodges d'après ses propres tableaux. Ce sont des portraits - en 1794 encore de la famille du stathouder; dans les années qui suivent, des sommités de la République batave telles que le général H.W. Daendels, l'amiral J.W. de Winter, et les présidents de l'Assemblée nationale P. Paulus et J.G.H. Hahn. Après les révolutionnaires néerlandais, ce sera bientôt le tour des militaires français : Bonaparte, Pichegru, Brune.

En ce qui concerne le contenu des estampes, Maaskamp commença à changer de cours juste après 1800. Deux allégories virent encore le jour, sur la Paix d'Amiens et sur le thème de la guerre, dessinées par J. Kuyper et "pointillées dans le meilleur style anglais" par L. Portman. Parurent ensuite deux reproductions par Hodges de tableaux du XVIIe siècle, le «Chef du Chantier Naval» de Rembrandt et la «Marchande de Harengs » de Gabriel Metsu; puis assez vite les premiers cahiers des Tableaux de l'habillement, avec des contributions entre autres de Portman, Kuyper et Pieneman; ensuite deux gravures par Van Os: « Le Taureau hollandais et les Brebis » et «Les Vaches hollandaises»; enfin la gravure du portrait de l'actrice Johanna Cornelia Ziesenis d'après un tableau de Hodges. De nouveau, Maaskamp avait engagé les artistes les plus doués d'Amsterdam. Jacques Kuyper (1761-1808), un des directeurs de l'École des Beaux-Arts de la ville,

(7) L'entreprise de Maaskamp et les différents aspects de son fonds font l'objet d'une thèse de doctorat qu'achève l'auteur du présent article. Elle aura pour titre De uitgever Evert Maaskamp en zijn fonds. Een bijdrage aan de geschiedenis van het Nederlandse nationaliteitsbesef.

(8) A. C. A. W. BARON VAN DER Feltz, Chanles Howard Hodges 1764-1837, Assen, 1982. R. VAN EIJNDEN et A. VAN DER WILLIGEN, Geschiedenis der vaderlandsche schilderkunst, Haarlem, 1816-1840, III, pp. 337-338 et IV, pp. 58-59. 
avait la réputation d'être un spécialiste dans le dessin des figures et des «ordonnances historiques » dans le style simple et élevé du néo-classicisme. Il travaillait le plus souvent avec le graveur Louis Portman (1772-ca.1829), un Allemand d'origine. Puis il y avait les jeunes peintres prometteurs, Pieter Gerardus van Os (1776-1839) et Jan Willem Pieneman (1779-1853); celui-ci deviendra célèbre grâce à sa "Bataille de Waterloo ", la grande pièce historique qui lui vaudra d'être décoré d'un ordre de chevalerie (9). À voir les gravures publiées par Maaskamp, il s'avère que prédominent désormais les thèmes culturels - et on a même l'impression qu'après la Paix d'Amiens, l'éditeur a complètement délaissé les thèmes politiques. Il n'en est rien. À l'inverse, son instinct infaillible semble lui avoir fait pressentir dans quel sens allait s'orienter la réalité politique.

\section{Une identité nationale}

Le comportement autoritaire de la France et la conscience d'une souveraineté bafouée et de la perte de son prestige, firent comprendre à la République batave le peu d'importance de son rôle sur la scène européenne. C'est là une des raisons pour lesquelles, vers 1800, les Pays-Bas se préoccupèrent de leur identité nationale plus que jamais auparavant. La nation néerlandaise, jadis célèbre dans le monde entier, qu'était-elle devenue? La question avait assez souvent été posée par des publicistes critiques bien avant 1800, mais elle semblait désormais être désagréablement d'actualité. Pour la première fois, on pressentait que le passé glorieux était vraiment à jamais disparu (10).

En même temps, ce passé devenait de plus en plus un sujet d'orgueil national. Le souvenir en était vivace. Des expressions comme "patrie» et « national », lourdement chargées maintenant d'un sens très émotif connurent un regain de vogue. Le passé - c'est-à-dire le XVIIe siècle -, glorifié en tant qu'histoire nationale, devait donner le courage d'affronter l'avenir. Cette conscience nationale - un mélange de défaitisme et d'espérance - est à la base de la formation de l'État-nation du début du XIXe siècle (11).

Et que fit Maaskamp dans tout cela? D'abord il contribua à raffermir la fierté nationale par les gravures qu'il éditait et qui pouvaient facilement soutenir la comparaison avec les éditions étrangères. Ensuite il les consacra

(9) Voir pour Pieneman, W. Loos (éd.), Een eeuw apart: Het Rijksmuseum en de Nederlandse schilderkunst in de 19de eeuw, Amsterdam, 1993, pp. 56-59. Sur Van Os, L. VAN TILBORG ET G. JANSEN, Op zoek naar de gouden eeuw. Nederlandse schilderkunst 1800-1850, Zwolle, 1986, pp. 139-143.

(10) N. C. F. VAN SAS, «Vaderlandsliefde, nationalisme en vaderlands gevoel», Tijdschrift voor Geschiedenis, 102, 1989, pp.471-495. Id., Talen van het vaderland. Over patriottisme en nationalisme, Amsterdam, 1995.

(11) Id, « De vaderlandse imperatief », op. cir. 
à des sujets dont la nation entière pouvait être fière: la meilleure peinture néerlandaise, les meilleurs bovins qui soient aux Pays-Bas et, ce qui fut sa contribution la plus originale : une nouvelle silhouette nationale par le biais de costumes régionaux, made in Nederland. Cette dernière contribution semble du reste être coupée sur le même patron que le dernier revirement politique - la grande variéte vestimentaire constitue pour ainsi dire le pendant culturel de l'opposition à l'unitarisme radical, mise en œuvre par la constitution de 1801 (12).

C'était un créneau commercial. Les Tableaux de l'Habillement de Maaskamp eurent un succès immédiat et la presse leur fit elle aussi un accueil enthousiaste. Tous les critiques louaient l'ouvrage pour sa nouveauté et sa qualité. Le soin avec lequel avaient été exécutées les estampes ne comptait pas pour rien dans leur appréciation. C'est que l'édition de Maaskamp ne faisait nullement penser aux gravures anciennes de costumes régionaux. Pour les Tableaux, une formule nouvelle avait été conçue, celle d'images «en action », comme le dit l'annonce. Les gravures ressemblent à de petits tableaux de genre. On y montre deux personnes en train de parler ou d'accomplir un acte quelconque - parfois avec l'intention évidente d'obtenir un effet comique. Les sous-titres nous permettent pour ainsi dire d'entendre ce qui y est dit, parfois même dans le patois local (illustration 1). Dans la perspective de la «nationalisation » à venir de la langue néerlandaise, l'accent mis sur les patois a malgré tout quelque chose de provoquant (13). C'est du reste à cet aspect narratif des gravures que réfère sans doute le terme "parlant» de la phrase "un tableau parlant de la patrie »; et à tout le moins à la vérité et à la ressemblance de l'ensemble.

\section{Un projet commun}

Pour la réalisation des Tableaux, Kuyper avait été chargé de la direction artistique. Pieneman faisait les dessins définitifs des esquisses de Kuyper, que Portman gravait ensuite sur la planche. Pieneman apposait les couleurs sur son dessin ou sur une épreuve servant de modèle à ceux qui coloriaient ensuite les gravures. Le texte de l'annonce - «imprimé en couleurs»-peut nous tromper, car les gravures étaient coloriées à la main. L'apport de Pieneman n'a pas été négligeable. Si nous regardons par exemple la composition de Kuyper pour la troisième estampe, «Je vous annonce la mort de ", il vaut mieux ne pas prendre trop au sérieux les mots «d'après nature » de l'annonce (illustration 4). L'image est supposée être celle d'une marchande de vin, un peu ivre, mais qui pose plutôt comme une danseuse,

(12) S. Schama, Patriots and Liberators, New York, 1977, pp. 410-433.

(13) Sur le néerlandais en tant que langue nationale, voir KLOEK et MuNHARDT, 1800. Blauwdrukken voor een samenleving, Den Haag, 2001, pp. 429-443. 


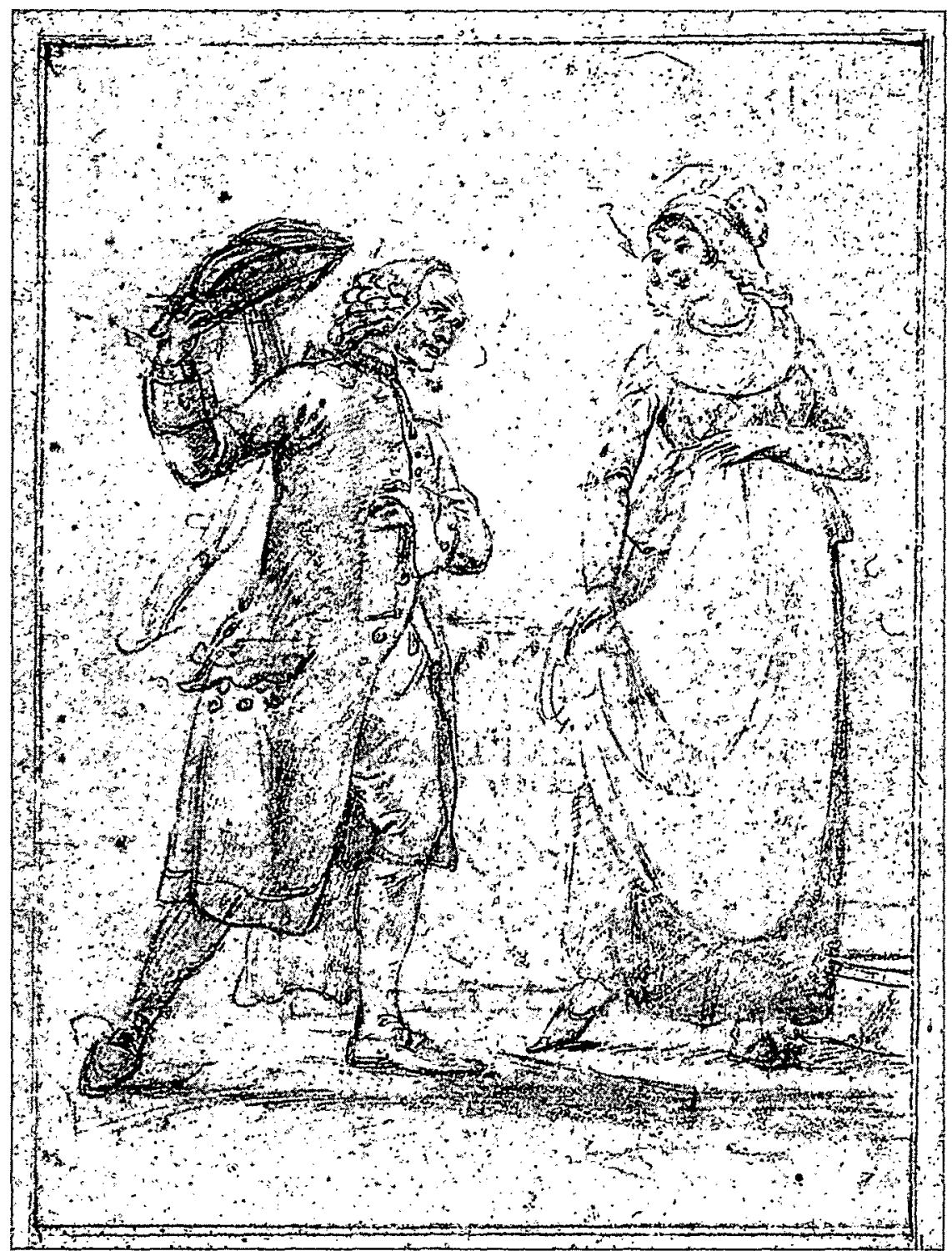

\section{Illustration 4}

J. Kuyper, esquisse au crayon pour illustration 5.

Rotterdam, Atlas van Stolk [5888-3a] 
avec une élégante tournure de la hanche et une jupe relevée. Pieneman lui fit subir une métamorphose complète. Sur l'estampe d'après son dessin, elle se tient tel le prototype de la ménagère hollandaise : les deux pieds sur terre, les mains sur les hanches (illustration 5).

Kuyper n'a pas eu à voyager dans tout le pays pour faire ses esquisses. Pour les provinces de Gueldre, de Brabant, quelques îles zélandaises et plusieurs lieux en Hollande et en Frise, des artistes étaient embauchés sur place. Dans ce sens aussi, c'était donc une véritable entreprise nationale. Derk van Oosterhout de Tiel, Quirinus van Amelsvoort de Bois-le-Duc, et Daniel de Keijzer de Middelbourg, ce sont là quelques noms d'artistes provinciaux qui ont contribué au projet. Ils devaient faire les recherches ethnographiques sur le terrain et noter exactement la coupe, les couleurs et les bijoux de l'habillement jusque dans les détails les plus minutieux. Pour les orienter dans leur travail, ils avaient devant eux l'esquisse de la composition de Kuyper. Les conceptions actuelles du métier d'artiste peuvent s'opposer à cette division du travail, mais ce procédé visait probablement à éviter de trop grandes ruptures stylistiques.

\section{Le costume traditionnel et le caractère national}

Comme nous l'avons vu, en 1805, la série des Tableaux était provisoirement achevée et pourvue d'une introduction. Dans ce texte, Maaskamp explique ce qu'il a eu en vue quand il a entrepris l'ouvrage. Il souhaitait que ses lecteurs fassent connaissance avec un aspect de la civilisation néerlandaise qui jusque-là n'avait jamais été bien exposé : "Il s'agit des divers costumes en usage parmi le peuple Batave, réunis à l'expression des caractères, des mœurs, des habitudes avec lesquels ces costumes ont une liaison intime » (14).

Car, selon Maaskamp, «le proverbe, qui dit que l'habit fait l'homme, peut bien souvent se prendre dans un sens inverse ». À moins que l'homme n'emploie ses habits comme un masque pour cacher sa nature véritable, son costume traduit son caractère. Et c'était là justement ce que désirait montrer Maaskamp. À l'aide des costumes régionaux, il voulait mettre en lumière les véritables caractères néerlandais - au pluriel, donc. Ce que le lecteur doit comprendre par là est le plus souvent passé sous silence.

L'estampe qui ouvre la série, «Une femme bourgeoise \& sa fille allant à l'église », en dit fort long sur ces intentions (illustration 6). Avec sa «cornette» de gaze noire, la mère est le parangon de «l'économie et de l'extrême propreté », attribuées aux femmes de la bourgeoisie hollandaise,

(14) E. MAASKAMP et J. KUYPER, Tableaux de l'habillement, des maurs et des coutumes dans la République batave au commencement du dix-neuvième siècle, Amsterdam, 1803-1807, introduction, s.p. 


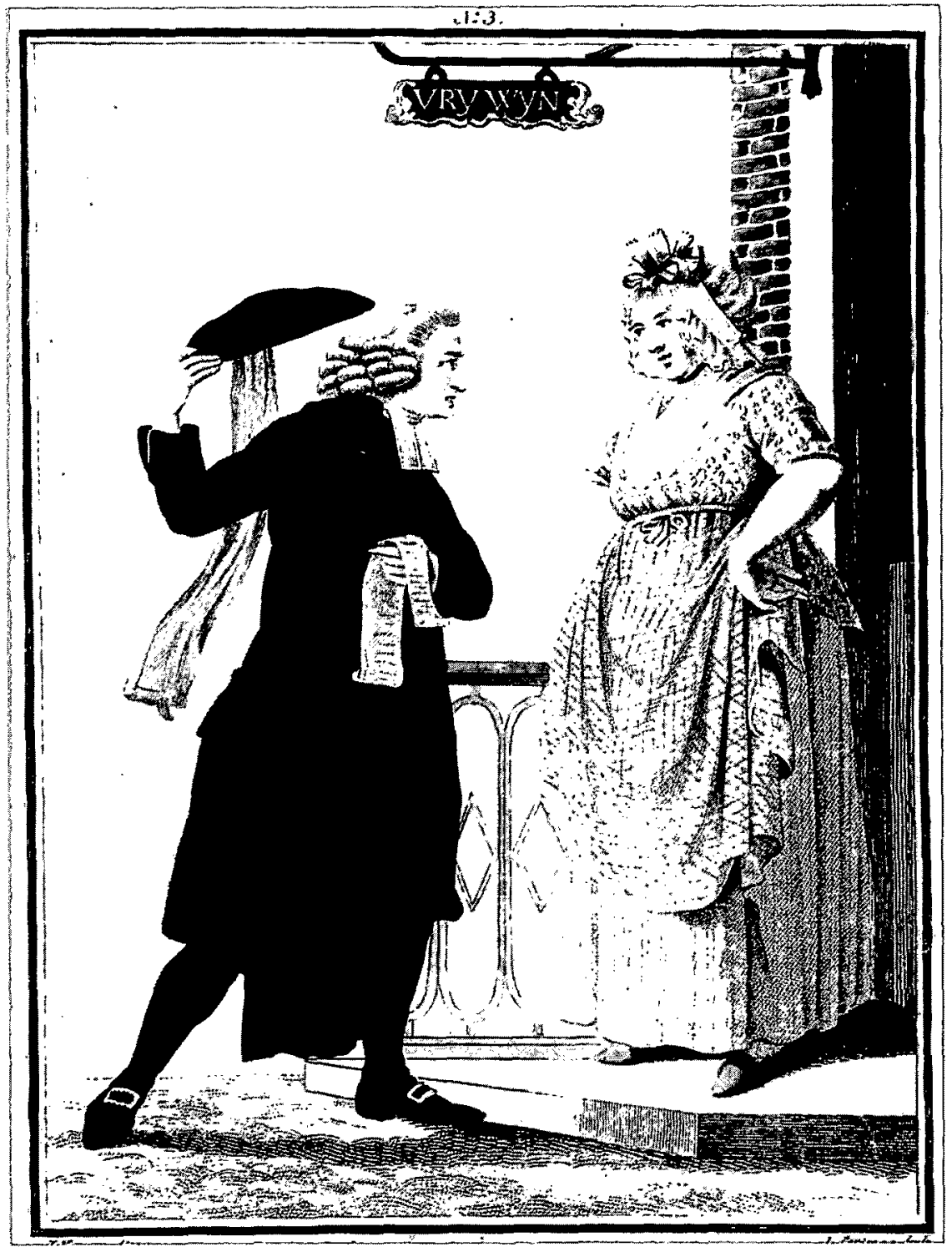

\section{Illustration 5}

J. W. Pieneman d'après J. Kuyper, dessins en couleurs pour l'estampe 3, "Amsterdamse aanspreker en tapster / Je vous annonce la mort de" Dans E. MAASKAMP et J. KUYPER, Tableaux de l'habillement [...], Amsterdam/Londen, 1803. 


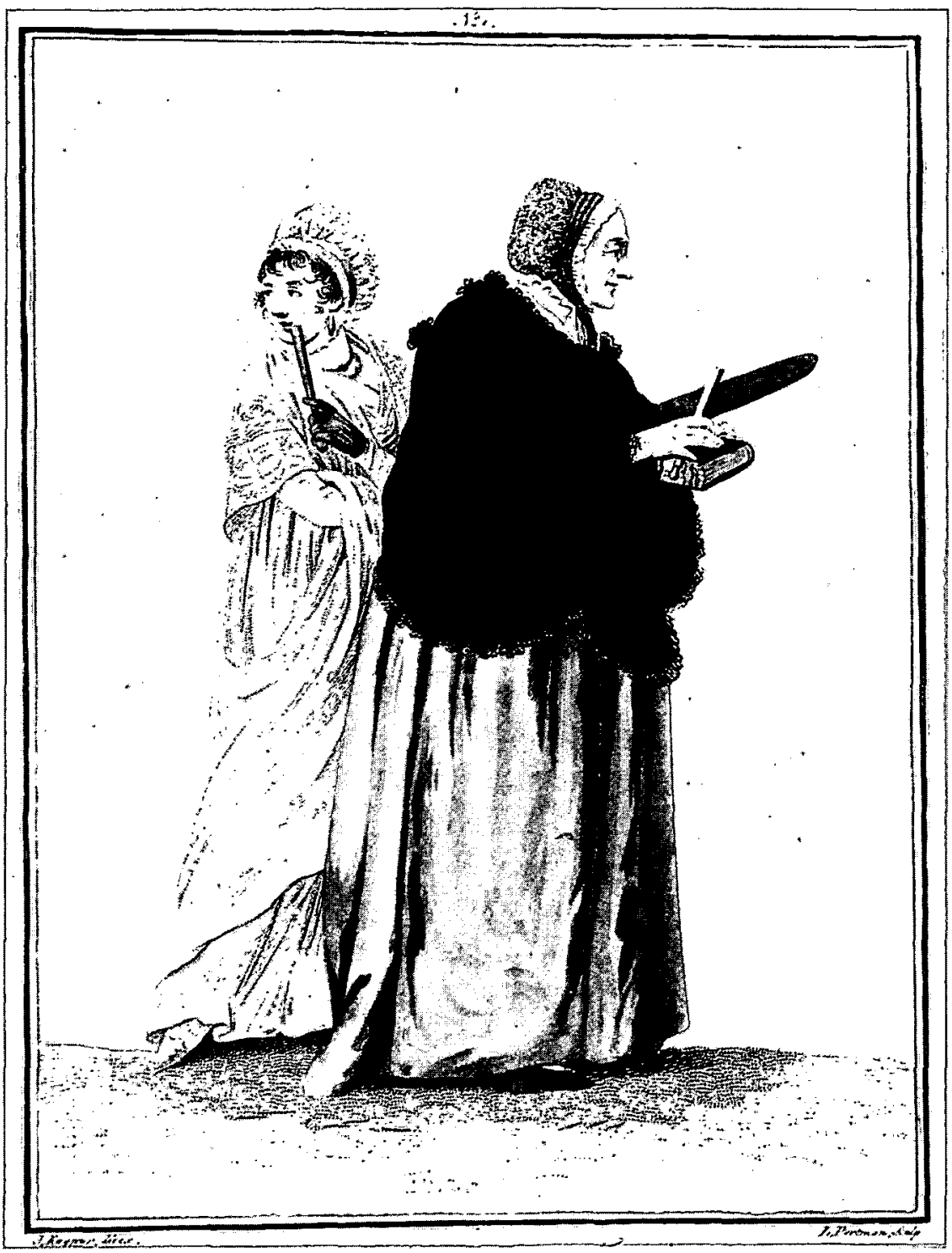

\section{Illustration 6}

L. Portman d'après J. Kuyper,

"Eene burger vrouw en dochter naar de kerk gaande. / Une femme bourgeoise et sa fille allant à l'église. " Dans E. MAASKAMP et J. KUYPER, Tableaux de l'habillement $[. .$.$] ,$ Amsterdam, 1803.

La Haye, Koninklijke Bibliotheek. [1374A98-pl.1] 
c'est ce que nous dit le texte accompagnant la gravure. La fille par contre est vêtue de la tête aux pieds selon la dernière mode. Elle a accru le volume de son bonnet, «pour faire paraître plus mignon le tour du visage ». Son regard et le signe qu'elle fait avec son éventail, trahissent que ses pensées sont loin d'être préoccupées par l'assistance au culte. Une telle légèreté, dit Maaskamp, est de tous les temps et de tous les pays. Autrement dit: elle n'est pas spécifique à un certain temps et à un certain lieu. Et c'est pourquoi il ajoute : « nous n'osons les donner comme des traits particuliers du caractère national au commencement du dix-neuvième Siècle " (15). Les explications accompagnant les autres costumes dévoilent à chaque fois que, pour Maaskamp, suivre la mode équivaut à trahir le caractère national et l'identité nationale. Aussi remarque-t-on deux poids et deux mesures. Le gros bonnet de la jeune fille est condamné, mais les chapeaux souvent démesurés des femmes revêtues du costume traditionnel trouvent malgré tout une place de choix dans l'ouvrage. Car, Maaskamp partait de l'idée que ce style s'était développé indépendamment de la mode, et qu'il était donc d'origine néerlandaise.

Pour ce qui est de sa vision de la mode, Maaskamp semble se trouver dans le camp des Cassandres de la culture. Mais, à leur encontre, il n'a pu avoir l'intention de critiquer sérieusement ses compatriotes friands de frivolités. Il s'en garda bien, car il aurait probablement choqué nombre de ses lecteurs. Du reste, alors que la série s'approchait de sa fin, la porte s'entrouvrit de nouveau pour laisser entrer sur scène des jeunes filles tout à fait à la mode. Ainsi sur la gravure «La promenade», finement dessinée par Kuyper, deux jeunes beautés de la Hollande septentrionale, sont complimentées pour leurs vêtements (illustration 7). Leurs costumes sont au goût du jour, comme le dit le texte, mais «sans artifices" et elles arborent la coiffe caractéristique de leur province. Quelques années plus tard, Maaskamp donnerait lui-même le ton dans le domaine de la mode, puisqu'en 1807 il créa le journal illustré Elegantia, d'après un modèle français.

\section{Une image exotique des Pays-Bas}

Une autre raison pourrait expliquer la dénonciation de la mode dans les Tableaux. C'est que Maaskamp voulait peindre dans cet ouvrage les Pays-Bas tel un pays extraordinaire, riche en particularités, comme nulle part ailleurs. Pour conforter cette image, il avait choisi un angle intéressant : l'extrême diversité culturelle. Selon lui, c'était de ce point de vue que les Pays-Bas se distinguaient des autres pays: «les diverses provinces de la République semblent être peuplées de nations différentes» (16). Pour

(15) Ibid, $\mathrm{n}^{\circ} 1$.

(16) Ibid., introduction et texte de l'annonce. 


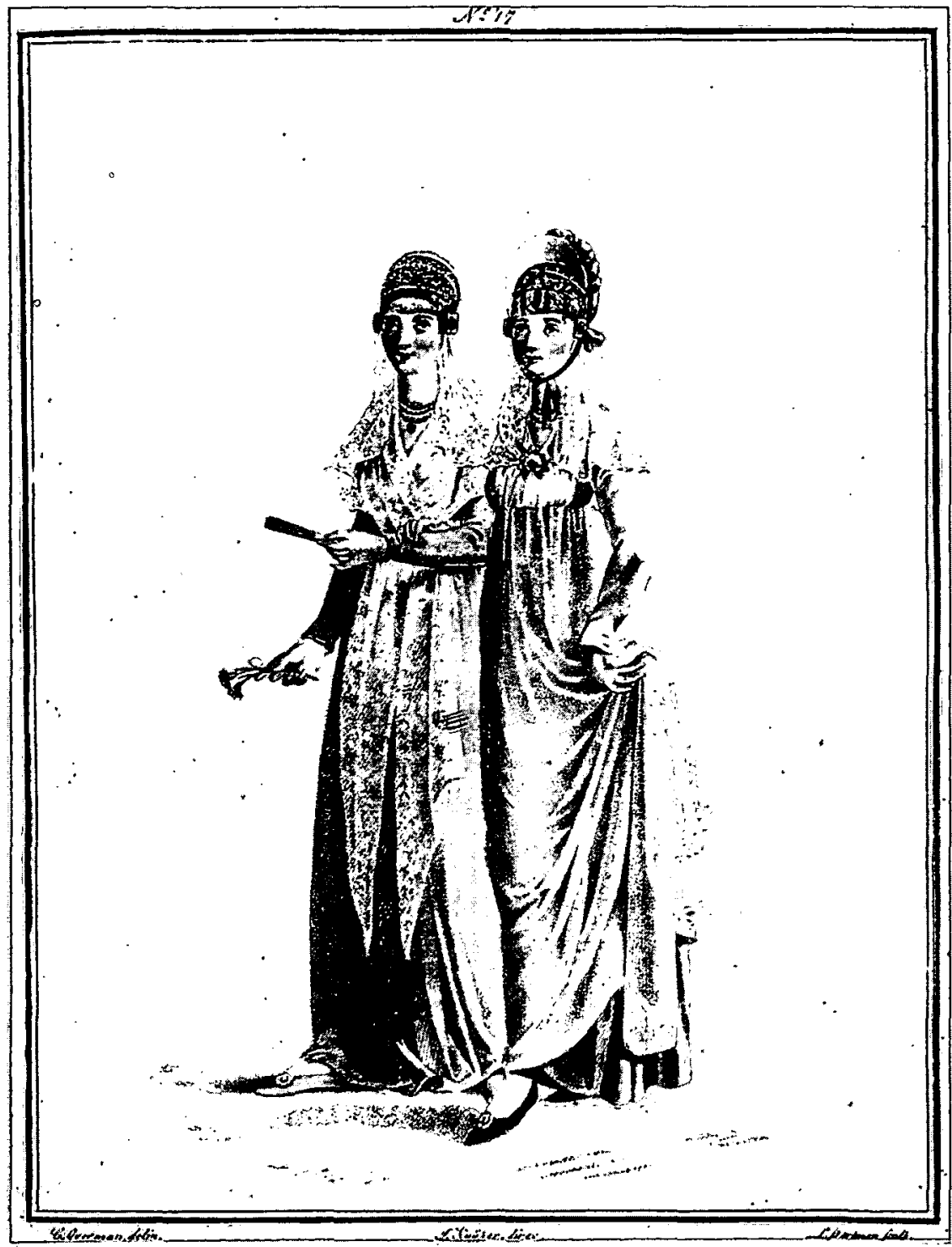

Illustration 7

L. Portman d'après J. Kuyper, « De wandeling / La promenade ». Dans E. MAASKAMP et J. KUYPER, Tableaux de l'habillement [...], Amsterdam/Londen, 1807. La Haye, Koninklijke Bibliotheek. 
donner relief à cette interprétation, Maaskamp pouvait difficilement utiliser des Néerlandais vêtus au dernier cri et parlant le néerlandais classique. Ils auraient troublé l'image. Aussi, seuls des paysans, des pêcheurs et des artisans pouvaient-ils poser pour son « tableau ».

Que l'identité néerlandaise soit précisément attribuée à ces "peuples particuliers" devait naturellement être expliqué. Maaskamp rechercha ses arguments dans deux disciplines qui jouissaient alors d'une grande autorité. C'étaient d'une part la théorie des climats, selon laquelle les conditions géographiques d'un territoire déterminent le caractère de ses habitants; d'autre part, l'archéologie du pays natal. Quant à la théorie des climats, la différentiation géographique de Maaskamp fait usage de mailles bien fines : un ruisseau ou une colline suffisent pour que diffèrent les cultures. Cela expliquerait pourquoi la Zélande compte presque autant de costumes que d'îles, tandis qu'il y aurait fort peu de costumes différents dans le Brabant ou la Gueldre. De plus, les hommes ressemblent un peu à leur environnement. Ainsi, les habitants de Walcheren : « la couleur même de leur habillement, paroît en général, empruntée des brouillards fréquents qui couvrent leur île » (17).

Si les hommes portant le costume régional étaient expliqués comme un phénomène naturel néerlandais, l'explication de la diversité du point de vue historique n'était pas moins évidente. Les costumes étaient liés aux différentes époques de notre histoire - dans le langage imagé de Maaskamp «ils étoient pour l'histoire autant de médailles ambulantes» (18). Leur ancienneté est pour lui une raison de plus pour sauvegarder les costumes régionaux. Ne serait-ce pas là de meilleurs témoins du passé que tous les récits historiques? C'est ainsi que, d'après Maaskamp, « les femmes de la Frise occidentale sont toujours [...] vêtues aussi bizarrement que l'étoient leurs ancêtres au cinquième siècle ", ceux-là mêmes qui fondaient des colonies en Grande-Bretagne (19). Quant aux habitants de Marken, ils n'auraient rien changé ni à leurs costumes ni à leurs usages depuis le XIIIe siècle quand l'île fut arrachée du continent par la tempête. Mais les titres les plus anciens reviennent au peuple de Schokland (illustration 8). Ici on peut toujours admirer l'original du portrait que Tacite avait peint des Bataves (20).

Il est impossible de savoir si les lecteurs de 1800 ont vraiment cru en l'inaltérabilité et en l'authenticité des costumes régionaux, ainsi que le croira plus tard le public (21). Mais dès sa parution les Tableaux libérèrent

(17) Ibid, $\mathrm{n}^{\circ} 13$.

(18) $\mathrm{Ibid}, \mathrm{n}^{\circ} 10$.

(19) Ibid., $\mathrm{n}^{\circ} 10$.

(20) Ibid, $\mathrm{n}^{\circ} 8$.

(21) Ce mythe qui trouve sans doute son origine chez J. le Francq van Berkhey a entre-temps été infirmé. Voir I. CIERAAD, De elitaire verheelding van volk en massa. Een studie over cultuur, Muiderberg, 1988. G. ROOIJAKKERS, "Dragers van traditie? Klederdracht als culturele constructie", dans D. VERHOEVEN et al. (ed.), Klederdracht en kleedgedrag. Het kostuum van Haner Majesteits onderdanen 1898-1998, Nijmegen, 1998, pp. 172-188. 


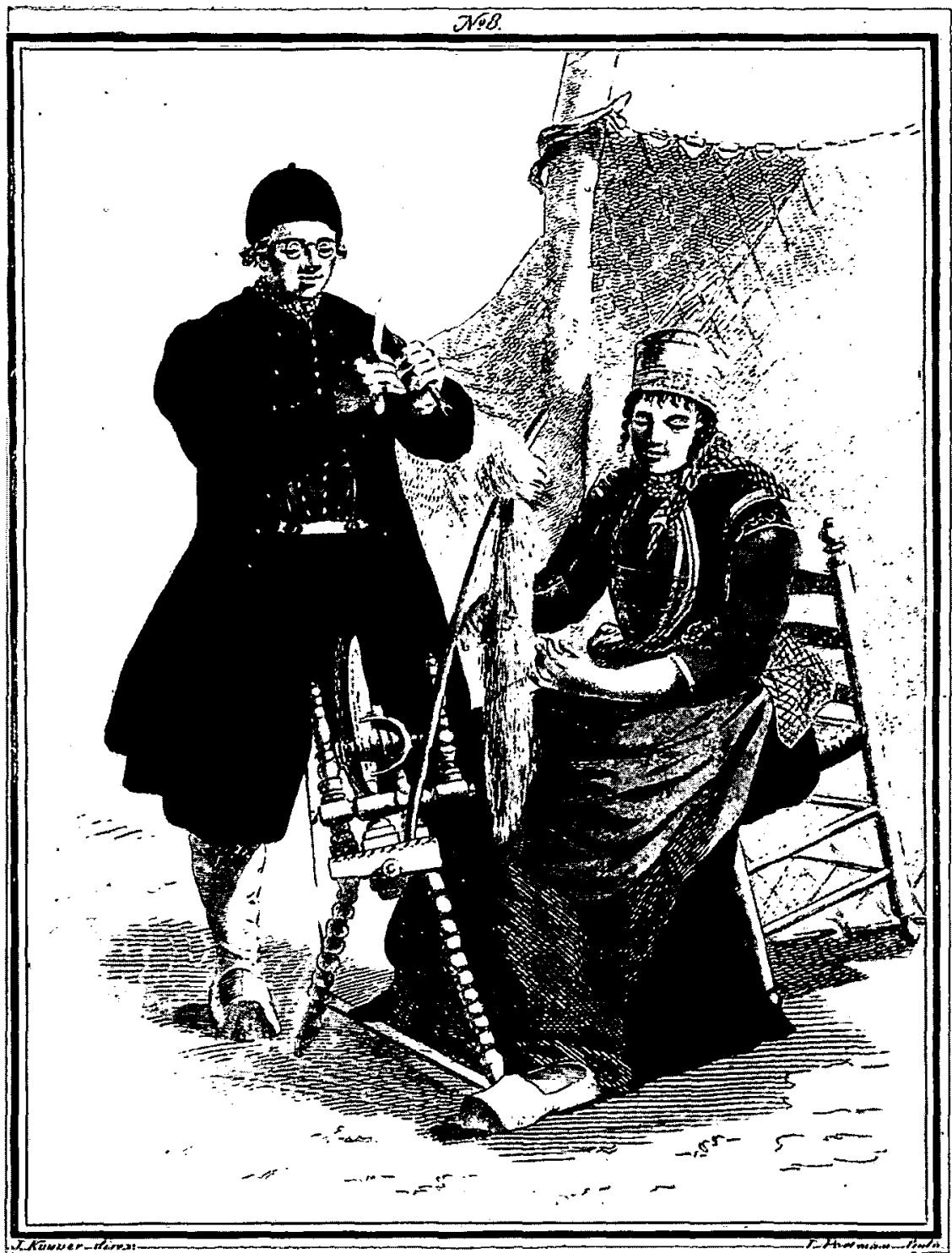

Illustration 8

L. Portman d'après J. Kuyper, « Kom vrouwtjen, spin af - het net is vol scheuren / Allons, file, file, ma femme - mes filets sont tous déchirés".

Dans E. MAASKAMP et J. KUYPER, Tableaux de l'habillement [...] A, Amsterdam/Londen, 1804. La Haye, Koninklijke Bibliotheek. 
des sentiments nationaux comme le démontre la réaction d'un critique qui reconnaît dans les costumes «le sérieux national » par lequel les Hollandais ont toujours inspiré le respect aux étrangers (22).

\section{Pour le bonheur des Hollandais}

L'accueil fait à l'étranger à la nouvelle image des Pays-Bas proposée par Maaskamp, constitue un sujet en soi, mais dans le contexte présent une réaction doit être mentionnée. F. J. B. baron D'Alphonse, depuis 1811, intendant de l'Intérieur en Hollande, prit de sa propre initiative la décision de faire un rapport ethnographique sur les Pays-Bas, qu'il a intégré dans son Aperçu sur la Hollande. D'Alphonse voulait donner un complément aux données statistiques et économiques que ses supérieurs lui avaient demandées (23). La méthode de recherche devait être plus ou moins celle qu'il avait suivie pour les données statistiques. Il demandait par circulaire des renseignements exacts concernant les mœurs et les habitudes des habitants à tous les préfets des départements hollandais (24). Cette approche professionnelle doit avoir plu aux Français, surtout dans les cercles diplomatiques, où l'on commençait à se rendre compte du fait que les connaissances disponibles sur la Hollande étaient en grande partie basées sur des idées préconçues et que les conséquences s'en faisaient sentir dans les relations entre les deux pays (25).

Ce n'est sans doute pas sans peine que les préfets et les sous-préfets ont recueilli des renseignements sur des choses personnelles comme «l'habillement et la coèffure des hommes et des femmes tant des villes que des campagnes ». Tant bien que mal ils ont couché par écrit leurs connaissances, traitant assidûment aussi bien de la ville que de la campagne. C'est justement pourquoi, du reste, la vue d'ensemble des costumes néerlandais qui émerge des réponses des préfets, est bien plus pâle que l'image peinte par Maaskamp. Toutes les réponses sur les costumes des citadins allaient dans le même sens; dans les grandes villes et dans les plus importantes des petites villes, seule une mode dominait en principe : la mode française (26).

(22) [Anonyme], Algemeene konst-en letterbode, 1806, II, p. 93.

(23) Aperçu sur la Hollande, présenté d Son Excellence le ministre de l'Intérieur par Mr. D'Alphonse, maître des requêtes au Conseil d'État, intendant de l'Intérieur en Hollande, Amsterdam 28 avril 1813. Algemeen Rijksarchief, Binnenlandse Zaken 1795-1813, nos 1129-1233. Les statistiques ont été publiées dans Centraal Bureau voor de Statistiek, n¹, La Haye, 1900. Sur D'Alphonse, voir Schama, op. cit., pp. 618-619.

(24) H.T. Colenbrander, Gedenkstukken, VI, pp. 1097-1109. Pour les réponses du préfet du Zuiderzee, J.R. PERSMAN, "Men is van mening dat Noord-Holland de mooiste vrouwen voortbrengt ", Holland, XI, 1979, $\mathrm{n}^{\circ} 1$, pp. 20-30.

(25) A. JOURDAN, "Les Gaulois en Batavie: des relations diplomatiques machiavéliques ", dans A. JOURDAN et J.TH. LEERSSEN, Remous révolutionnaines. République batave, armée française, Amsterdam, 1996, pp. 91-118.

(26) Sur ces données d'archives, cf ma thèse (note 7). 
Dans l'Aperçu ne se retrouve qu'une fraction des données sur l'habillement, rassemblées avec tant de zèle. Apparemment, D'Alphonse avait trouvé mieux avant de commencer son rapport. Enchanté, le voilà qui écrit en effet : « Ce serait un tableau curieux que celui qui tracerait avec fidélité la variété de tous les costumes » (27). Et d'ajouter «Il paraît qu'on l'a déjà senti », après quoi il signale les fameux Tableaux de Maaskamp, qui étaient évidemment bien supérieurs aux descriptions des préfets. D'Alphonse mit donc de côté celles-ci et choisit de faire parvenir en France non pas l'image la plus complète, mais la plus caractéristique de l'habillement hollandais.

En 1813, les gravures de Maaskamp furent envoyées à Paris en supplément au chapitre sur les costumes de l'Aperçu. Les gravures des costumes zélandais faisaient partie de l'envoi, bien que la Zélande eût déjà été détachée de la Hollande. D'Alphonse n'y ajouta pas les explications imprimées; il les recopiait partiellement, et souvent littéralement. Mais le brave homme en profitait pour ôter prudemment les remarques piquantes, telle celle sur un collier en corail «dont le rouge tranche admirablement sur une gorge d'albâtre " (28). Et il laissait aussi de côté les citadins qui s'habillaient à la mode. Aussi peut-il écrire, tout comme Maaskamp : «Il semble que ce n'est pas la même nation mais un composé de nations différentes 》 (29).

Dans le chapitre sur le caractère des Néerlandais aussi, D'Alphonse insiste sur la remarquable diversité culturelle des départements hollandais, ce qui témoigne que l'image spécifique qu'il avait choisie de donner des Pays-Bas avait en outre une raison politique. C'était là un effort touchant pour inciter Napoléon à tenir compte des particularités du pays et de son peuple. Dans ce contexte, la série des costumes de Maaskamp, avec sa représentation pénétrante de certaines singularités hollandaises, pouvait pour ainsi dire remplir une fonction diplomatique.

Dans le passé, écrit D'Alphonse, Charlemagne a su imposer aux anciens Frisons sa culture et ses lois sans nuire à leur bonheur :

«Pourquoi ne serait-il pas possible à l'empereur après avoir fixé les destinées du monde, après avoir triomphé de toute résistance, d'assurer aussi le bonheur des hollandais. Instruit du vrai caractère des habitans de cette partie petite mais intéressante de son Empire, de leur situation et de leurs besoins, leur bonheur ne dépendra que d'un acte de sa volonté [...] Qu'il est doux de songer ainsi à l'avenir ! »

(Traduction du néerlandais : A. Kost et A. Jourdan)

Eveline KoOlHAAS-Grosfeld Université d'Amsterdam

Velhorst 5

N. L. -7241 TB Lochem

(27) D'ALPHONSE, op. cit., 1232, pp. 214-215.

(28) MAASKAMP \& KUYPER, op. cit., $n^{\circ} 4$.

(29) D'ALPHONSE, op. cit., pp. 214-215. 